\title{
Density Distribution in Walsh Transform Sectors as Feature Vectors for Image Retrieval
}

\author{
H.B.Kekre \\ Sr. Professor \\ MPSTME, SVKM's NMIMS (Deemed-to be- \\ University) \\ Vile Parle West, Mumbai -56,INDIA
}

\author{
Dhirendra Mishra \\ Assistant Professor \& PhD Research Scholar \\ MPSTME, SVKM's NMIMS (Deemed-to be- \\ University) \\ Vile Parle West, Mumbai -56,INDIA
}

\begin{abstract}
This paper presents the idea of using sal cal density distribution in complex Walsh transform sectors to generate the feature vector for content based image retrieval This paper compares the performance of 8,12 and 16 sectors of Walsh Transform. The density distribution of real (sal) and imaginary (cal) values of Walsh sectors in all three color planes are considered to design the feature vector. The algorithm proposed here is worked over database of 270 images spread over 11 different classes. The Euclidean distance is used as similarity measure. Overall Average precision and recall is calculated for the performance evaluation and comparison of $8,12 \& 16$ Walsh sectors. The overall average of cross over points of precision and recall is of all methods are compared.
\end{abstract}

\section{General Terms}

CBIR, Walsh Transform, Kekre's Algorithm

\section{Keywords}

CBIR,Walsh Transform, Euclidian Distance, Precision, Recall

\section{INTRODUCTION}

The earliest use of the term content-based image retrieval in the literature seems to have been by Kato [2], to describe his experiments into automatic retrieval of images from a database by color, texture and shape features. The term has since been widely used to describe the process of retrieving desired images from a large collection on the basis of features (such as colors, texture and shape) that can be automatically extracted from the images themselves. The typical CBIR system [1-6] performs two major tasks. The first one is feature extraction (FE), where a set of features, called image signature or feature vector, is generated to accurately represent the content of each image in the database. A feature vector is much smaller in size than the original image, typically of the order of hundreds of elements (rather than millions). The second task is similarity measurement (SM), where a distance between the query image and each image in the database using their signatures is computed so that the top —closest images can be retrieved.[79].

\section{WALSH TRANSFORM}

Walsh transform [15] matrix is defined as a set of $\mathrm{N}$ rows, denoted $\mathrm{Wj}$, for $\mathrm{j}=0,1, \ldots, \mathrm{N}-1$, which have the following properties:
- $\quad \mathrm{Wj}$ takes on the values +1 and -1 .

- $\quad W j[0]=1$ for all $\mathrm{j}$.

- $\quad \mathrm{Wj}_{\mathrm{x} \mathrm{W}} \mathrm{W}_{\mathrm{k}}^{\mathrm{T}}=0$, for $\mathrm{j} \neq \mathrm{k}$ and $\mathrm{Wj} \times \mathrm{Wk}^{\mathrm{T}}=\mathrm{N}$, for $\mathrm{j}=\mathrm{k}$.

- $\quad W j$ has exactly $\mathrm{j}$ zero crossings, for $\mathrm{j}=0,1, ., \mathrm{N}-1$.

- Each row $\mathrm{Wj}$ is either even or odd with respect to its midpoint.

Walsh transform matrix is defined using a Hadamard matrix of order N. The Walsh transform matrix row is the row of the Hadamard matrix specified by the Walsh code index, which must be an integer in the range $[0, \ldots, \mathrm{N}-1]$. For the Walsh code index equal to an integer $\mathrm{j}$, the respective Hadamard output code has exactly $\mathrm{j}$ zero crossings, for $\mathrm{j}=0,1, \ldots, \mathrm{N}-1$.

Kekre's Algorithm to generate Walsh Transform from Hadamard matrix [16]:

Step 1:

Arrange the ' $n$ ' coefficients in a row and then split the row in ' $\mathrm{n} / 2$ ', the other part is written below the upper row but in reverse order as follows:

$\begin{array}{llllllllllllllll}0 & 1 & 2 & 3 & 4 & 5 & 6 & 7 & 8 & 9 & 10 & 11 & 12 & 13 & 14 & 15\end{array}$

15141312111098

Step 2:

We get two rows, each of this row is again split in ' $n / 2$ ' and other part is written in reverse order below the upper rows as shown below.

$\begin{array}{llll}0 & 1 & 2 & 3\end{array}$

15141312

$\begin{array}{llll}7 & 6 & 5 & 4\end{array}$

$\begin{array}{llll}8 & 9 & 10 & 11\end{array}$

This step is repeated until we get a single column which gives the ordering of the Hadamard rows according to sequency as given below:

$0,15,7,8,3,12,4,11,1,14,6,9,2,13,5,10$

Step 3:

According to this sequence the Hadamard rows are arranged to get Walsh transform matrix. Now a product of Walsh matrix 
and the image matrix is calculated. This matrix contains Walsh transform of all the columns of the given image.

Since Walsh matrix has the entries either +1 or -1 there is no multiplication involved in computing this matrix. Since only additions are involved computation complexity is very low.

\section{FEATURE VECTOR GENERATION}

The proposed algorithm makes novel use of Walsh transform to design the sectors to generate the feature vectors for the purpose of search and retrieval of database images. The complex Walsh transform is conceived by multiplying all sal functions by $\mathrm{j}$ and combining them with real cal functions of the same sequency. Thus it is possible to calculate the angle by taking tan inverse of sal/cal. However the values of tan are periodic with the period $\Pi$ radians hence it can resolve these values in only two sectors. To get the angle in the range of 0 360 degrees we divide these points in four sectors as explained below. These four sectors are further divided into 8,12 and 16 sectors. .The density distribution of sal and cal in each sector is considered for feature vector generation. Each Walsh sector is represented by single percentage value of sal cal distribution in particular sector w.r.t. all sectors for feature vector generation; calculated as follows

(Total number of sal in particular sector) X 100

(Total number of sal in all sectors)

Thus for 8 and $12 \& 16$ Walsh sectors 8 and 12 feature components for each color planes i.e. R, G and B are generated. In all feature vector is of dimension 24, 36 and 48 components.

\subsection{Four Walsh Transform Sectors:}

To get the angle in the range of 0-360 degrees, the steps as given in Table 1 are followed to separate these points into four quadrants of the complex plane. The Walsh transform of the color image is calculated in all three R, G and B planes. The complex rows representing sal components of the image and the real rows representing cal components are checked for positive and negative signs. The sal and cal Walsh values are assigned to each quadrant. as follows:

Table 1. Four Walsh Sector formation

\begin{tabular}{|c|c|c|}
\hline $\begin{array}{l}\text { Sign of } \\
\text { Sal }\end{array}$ & $\begin{array}{l}\text { Sign of } \\
\text { Cal }\end{array}$ & Quadrant Assigned \\
\hline+ & + & I (0-90 Degrees $)$ \\
\hline+ & - & II ( $90-180$ Degrees $)$ \\
\hline- & - & III( 180- 270 Degrees $)$ \\
\hline- & + & IV(270-360 Degrees) \\
\hline
\end{tabular}

The equation (1) is used to generate individual components to generate the feature vector of dimension 12 considering three $\mathrm{R}, \mathrm{G}$ and $\mathrm{B}$ Planes. The sal and cal density distribution in all sectors is used for feature vector generation. However, it is observed that the density variation in 4 quadrants is very small for all the images. Thus the feature vectors have poor discretionary power and hence higher number of sectors such as 8,12 and 16 were tried. Euclidean distance measure is used to check the closeness of the query image from the database image and precision recall are calculated to measure the overall performance of the algorithm.

\subsection{Eight Walsh Transform Sectors:}

Each quadrants formed in the previous obtained 4 sectors are individually divided into 2 sectors each considering the angle of 45 degree. In total we form 8 sectors for R,G and B planes separately as shown in the Table 2 . The percentage density distribution of sal and cal in all 8 sectors are determined using equation (1) to generate the feature vector.

Table 2. Eight Walsh Sector formation

\begin{tabular}{|c|c|c|}
\hline $\begin{array}{l}\text { Quadrant of } 4 \\
\text { Walsh sectors }\end{array}$ & Condition & New sectors Formed \\
\hline \multirow[t]{2}{*}{$\mathrm{I}\left(0-90^{0}\right)$} & $\mathrm{Cal}>=\mathrm{Sal}$ & I (0-45 Degrees) \\
\hline & $\mathrm{Sal}>\mathrm{Cal}$ & II (45-90 Degrees) \\
\hline \multirow[t]{2}{*}{ II $\left(90-180^{\circ}\right)$} & Sal | > |Cal & III(90-135 Degrees) \\
\hline & $|\mathrm{Cal}|>=\mid$ Sal $\mid$ & IV(135-180 Degrees) \\
\hline \multirow[t]{2}{*}{ III $\left(180-270^{0}\right)$} & $|\mathrm{Cal}|>=\mid$ Sal $\mid$ & V (180-225 Degrees ) \\
\hline & $\mid$ Sal $|>| \mathrm{Cal} \mid$ & VI (225-270 Degrees) \\
\hline \multirow[t]{2}{*}{ IV $\left(270-360^{0}\right)$} & $\mid$ Sal $|>| \mathrm{Cal} \mid$ & VII (270-315 Degrees) \\
\hline & $|\mathrm{Cal}|>=|\mathrm{Sal}|$ & VIII (315-360 Degrees ) \\
\hline
\end{tabular}

\subsection{Twelve Walsh Transform Sectors:}

Each quadrants formed in the previous section of 4 sectors are individually divided into 3 sectors each considering the angle of 30 degree. In total we form 12 sectors for R,G and B planes separately as shown in the Table 3 . The percentage density distribution of sal and cal in all 12 sectors are determined using equation (1) to generate the feature vector

Table 3. Twelve Walsh Sector formation

\begin{tabular}{|c|c|c|}
\hline 4 Quadrants & Condition & New sectors \\
\hline \multirow[t]{3}{*}{$\mathrm{I}\left(0-90^{0}\right)$} & Cal $>=\sqrt{3} *$ Sal & $\mathrm{I}\left(0-30^{0}\right)$ \\
\hline & $1 / \sqrt{3} \mathrm{cal}<=\mathrm{sal}<=\sqrt{3} \mathrm{cal}$ & II $\left(30-60^{0}\right)$ \\
\hline & Otherwise & III $\left(60-90^{0}\right)$ \\
\hline \multirow[t]{3}{*}{ II $\left(90-180^{0}\right)$} & $\mathrm{Cal}>=\sqrt{3} * \mathrm{Sal}$ & IV $\left(90-120^{0}\right)$ \\
\hline & $\begin{array}{c}1 / \sqrt{3} \mid \text { cal }|<=| \text { sal } \mid<=\sqrt{3} \\
\mid \text { cal } \mid\end{array}$ & $\mathrm{V}\left(120-150^{0}\right)$ \\
\hline & Otherwise & VI $\left(150-180^{0}\right)$ \\
\hline \multirow[t]{3}{*}{ III $\left(180-270^{0}\right)$} & $|\mathrm{Cal}|>=\sqrt{3} *|\mathrm{Sal}|$ & VII $\left(180-210^{0}\right)$ \\
\hline & $\begin{array}{c}1 / \sqrt{3} \text { cal }<=\mid \text { sal } \mid<=\sqrt{3} \\
\mid \text { cal } \mid\end{array}$ & VIII $\left(210-240^{0}\right)$ \\
\hline & Otherwise & IX $\left(240-270^{0}\right)$ \\
\hline $\begin{array}{l}\text { IV }(270- \\
\left.360^{\circ}\right)\end{array}$ & $|\mathrm{Cal}|>=\sqrt{3} *|\mathrm{Sal}|$ & $\mathrm{X}\left(270-300^{0}\right)$ \\
\hline
\end{tabular}




\begin{tabular}{|c|c|c|}
\hline & $\begin{array}{c}1 / \sqrt{3} \mid \text { cal }|<=| \text { sal } \mid<=\sqrt{3} \\
\mid \text { cal } \mid\end{array}$ & XI $\left(300-330^{\circ}\right)$ \\
\hline & Otherwise & XII $\left(330-360^{\circ}\right)$ \\
\hline
\end{tabular}

\section{RESULTS AND DISCUSSION}

The sample Images of the database of 270 images of 11 different classes such as Flower, Sunset, Barbie, Tribal, Puppy, Cartoon, Elephant, Dinosaur, Bus, Parrots, Scenery is shown in the Fig 1.
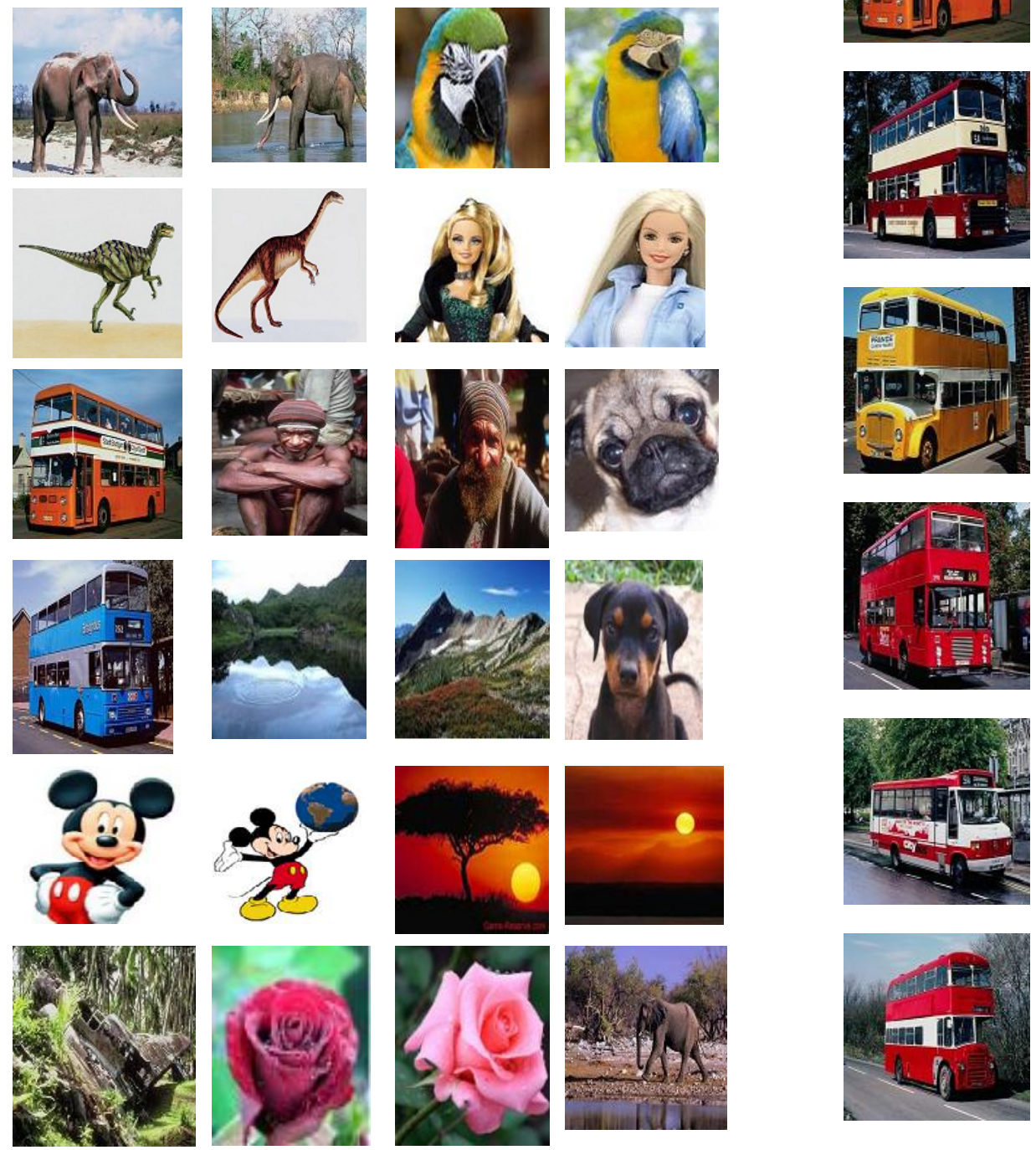

Fig.1 Sample Image Database

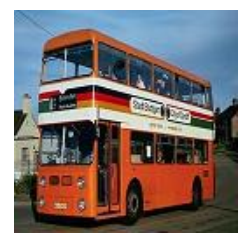

Fig. 2 Query Image

The image of the class Bus is taken as sample query image as shown in the Fig. 2. The first 18 images retrieved in the case of
12 Walsh sector used for feature vectors is shown in the Fig, 3. It is very clearly visible from the result that this approach gives the best result of retrieval. It is seen that only two images of irrelevant class are retrieved among first 18 images and rest are of query image class i.e. Bus.
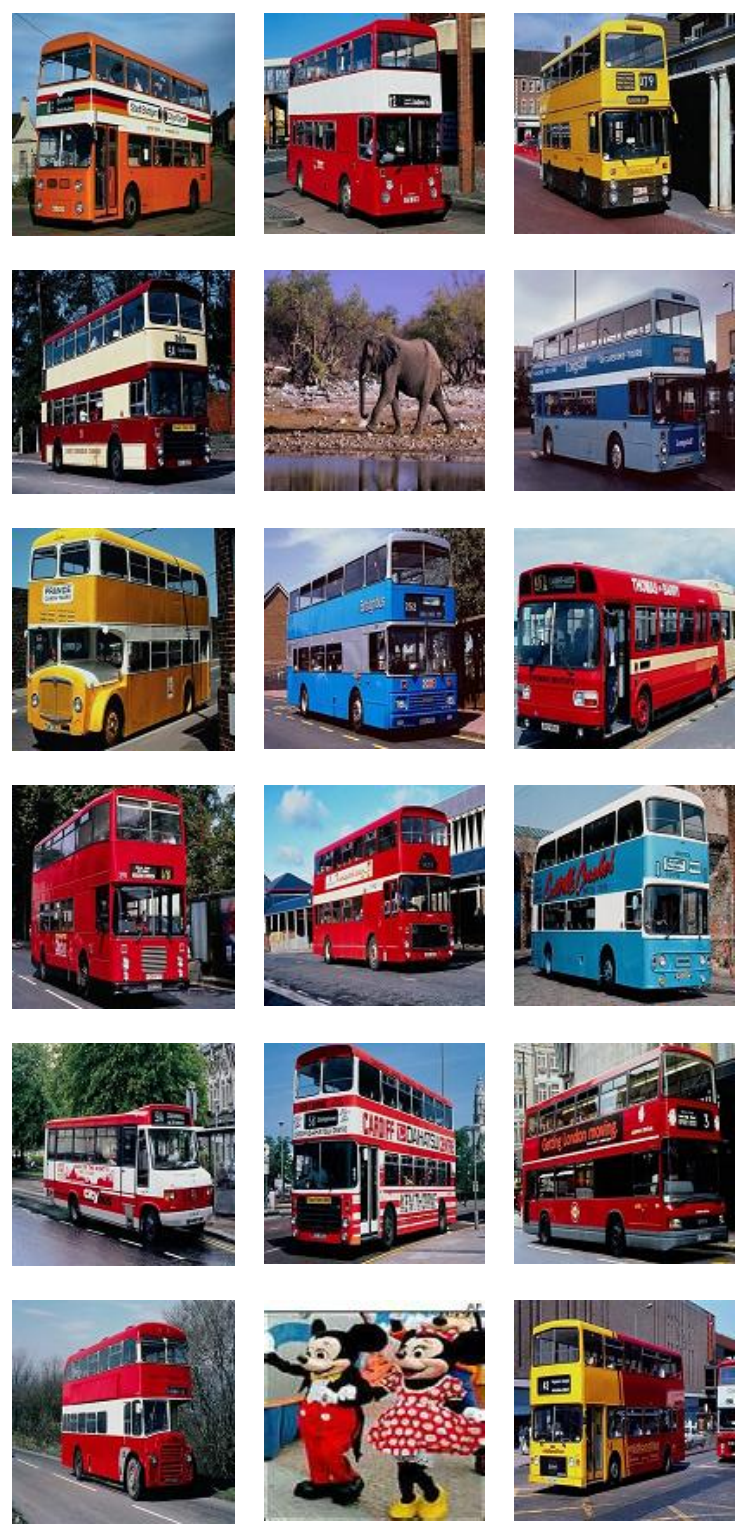

Fig. 3: First 20 Retrieved Images for the query image shown in the Fig. 2.

Once the feature vector is generated for all images in the database a feature database is created. A query image of each class is produced to search the database. The image with exact match gives minimum Euclidean distance. To check the effectiveness of the work and its performance with respect to retrieval of the images we have calculated the precision and recall as given in Equations (2) \& (3) below: 
Number of relevant images retrieved

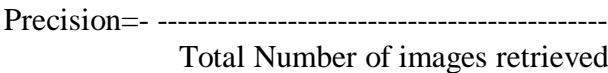

$$
\text { Recall= } \frac{\text { Number of Relevant images retrieved }}{\text { Total number of relevant images in database }}
$$

The Fig. 4 - Fig. 6 shows the overall average precision and recall performance of Walsh 8, 12 and 16 sectors respectively. The cross over points of overall average of precision and recall for 8, 12 and 16 sectors is shown in the Fig.7. It is observed that performance of 12 sectors is the best.

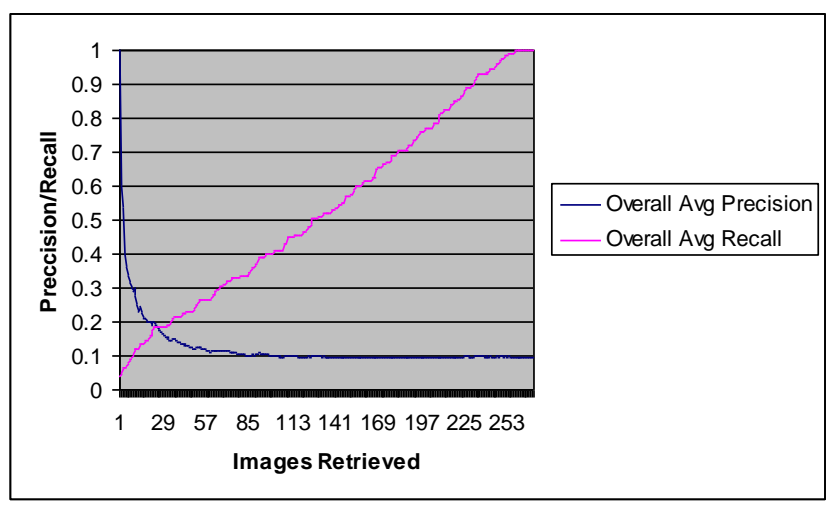

Fig 4 Overall Average Precision and Recall performance of density in 8 Walsh Transform sectors.

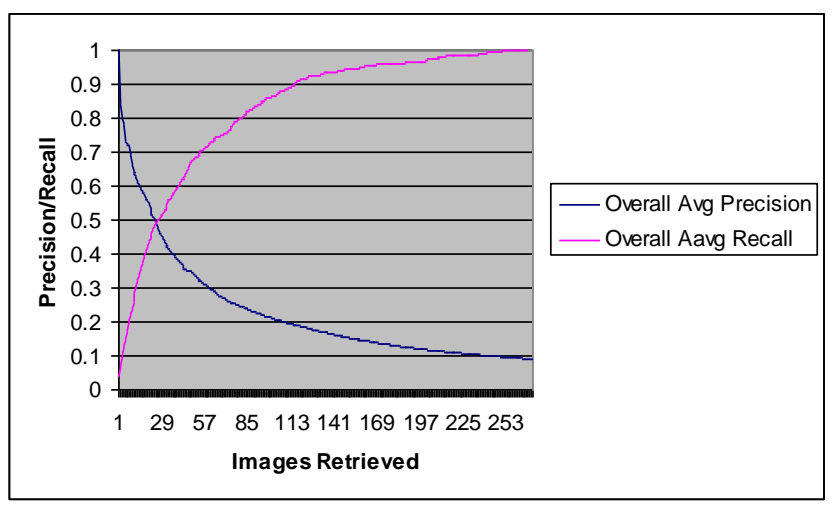

Fig 5: Overall Average Precision and Recall performance of density in 12 Walsh Transform sectors.

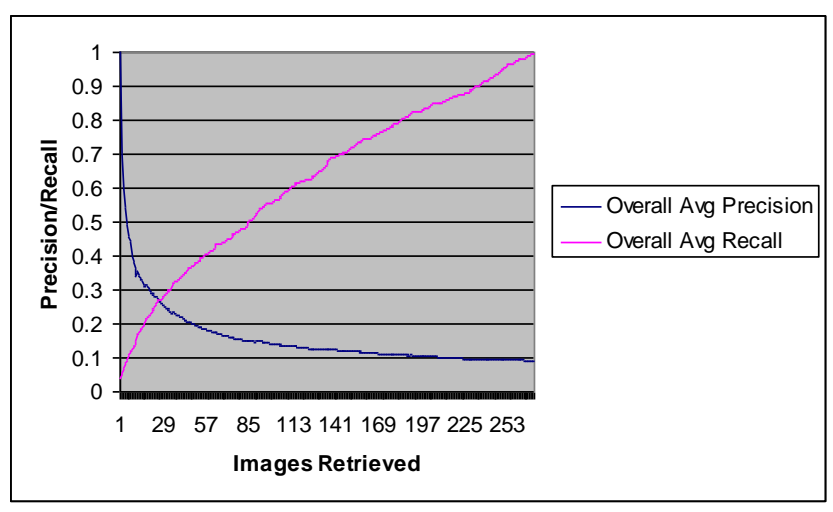

Fig 6 Overall Average Precision and Recall performance of density in 16 Walsh Transform sectors.

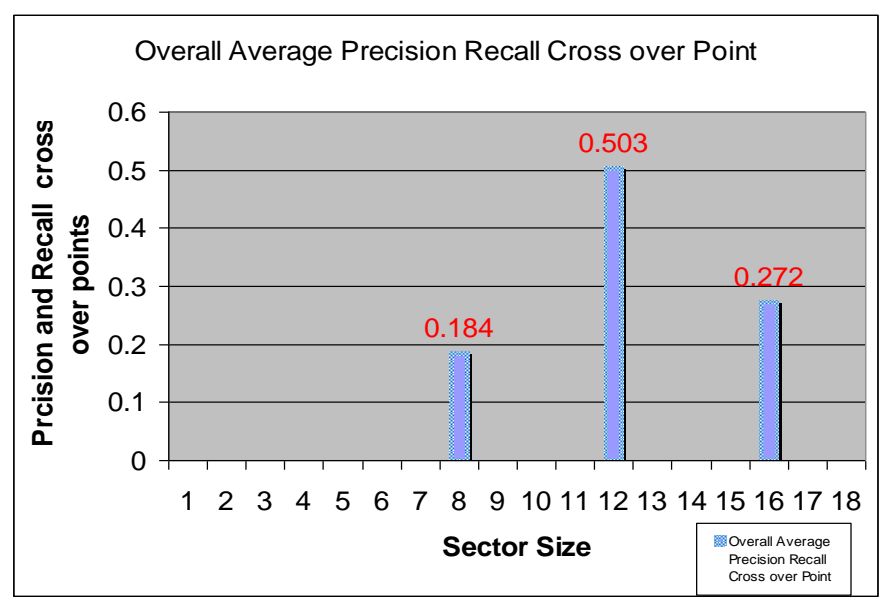

Fig. 7: Comparison of Precision and Recall cross over points based on sal cal density in Walsh 8,12 and 16 sectors

\section{CONCLUSION}

The Innovative idea of using complex Walsh transform 8,12 and 16 sectors of the images to generate the feature vectors for the purpose of image search and retrieval i.e. content based image retrieval is proposed. Using Walsh transform reduces the computational complexity reducing the search time to less than 5 seconds per query image. The performance of 8,12 and 16 sectors is compared. It is found that 12 sectors give the best result followed by 16 and 8.12 sectors give the best outcome of overall average precision and recall cross over point as 0.503 and it is 0.184 and 0.272 for 8 and 16 sectors respectively as shown in the Fig. 7. The proposed algorithm was experimented on a database of 270 images having 11 different classes. The Overall average precision and recall cross over points of all three approaches are plotted as shown in Fig 4- Fig 6

\section{REFERENCES}

[1] Anil Jain, Arun Ross, Salil Prabhakar, "Fingerprint matching using minutiae and texture features," Int'l conference on Image Processing (ICIP), pp. 282-285, Oct. 2001. 
[2] Kato, T., "Database architecture for contentbasedimage retrieval in Image Storage and Retrieval Systems" (Jambardino A and Niblack W eds),Proc SPIE 2185, pp 112-123, 1992.

[3] John Berry and David A. Stoney "The history and development of fingerprinting," in Advances in Fingerprint Technology, Henry C. Lee and R. E. Gaensslen, Eds., pp. 1-40. CRC Press Florida, $2^{\text {nd }}$ edition, 2001.

[4 ] Emma Newham, "The biometric report," SJB Services, 1995.

[5] H. B. Kekre, Dhirendra Mishra, "Digital Image Search \& Retrieval using FFT Sectors" published in proceedings of National/Asia pacific conference on Information communication and technology(NCICT 10) $5^{\mathrm{TH}} \& 6^{\mathrm{TH}}$ March 2010.SVKM'S NMIMS MUMBAI

[ 6 ] H.B.Kekre, Dhirendra Mishra, "Content Based Image Retrieval using Weighted Hamming Distance Image hash Value" published in the proceedings of international conference on contours of computing technology pp. 305-309 (Thinkquest2010) 13 th $\& 14^{\text {th }}$ March 2010.

[ 7 ] H.B.Kekre, Dhirendra Mishra, "Digital Image Search \& Retrieval using FFT Sectors of Color Images" published in International Journal of Computer Science and Engineering (IJCSE) Vol. 02,No.02,2010,pp.368-372 ISSN 0975-3397 available online at http://www.enggjournals.com/ijcse/doc/IJCSE10-02$\underline{02-46 . p d f}$

[ 8 ] H.B.Kekre, Dhirendra Mishra, "CBIR using upper six FFT Sectors of Color Images for feature vector generation" published in International Journal of Engineering and Technology(IJET) Vol. 02, No. 02, 2010, 49-54 ISSN 0975-4024 available online at http://www.enggjournals.com/ijet/doc/IJET10-0202-06.pdf

[9] Arun Ross, Anil Jain, James Reisman, "A hybrid fingerprint matcher," Int'l conference on Pattern Recognition (ICPR), Aug 2002.

[ 10 ] A. M. Bazen, G. T. B.Verwaaijen, S. H. Gerez, L. P. J. Veelenturf, and B. J. van der Zwaag, "A correlation-based fingerprint verification system," Proceedings of the ProRISC2000 Workshop on Circuits, Systems and Signal Processing, Veldhoven, Netherlands, Nov 2000.

[ 11] H.B.Kekre, Tanuja K. Sarode, Sudeep D. Thepade, "Image Retrieval using Color-Texture Features from DCT on VQ Codevectors obtained by Kekre's Fast Codebook Generation", ICGST International Journal on Graphics, Vision and Image Processing (GVIP), Available online at http://www.icgst.com/gvip

[ 12 ] H.B.Kekre, Sudeep D. Thepade, "Using YUV Color Space to Hoist the Performance of Block Truncation Coding for Image Retrieval", IEEE International Advanced Computing Conference 2009 (IACC'09), Thapar University, Patiala, INDIA, 6-7 March 2009.
[ 13 ] H.B.Kekre, Sudeep D. Thepade, "Image Retrieval using Augmented Block Truncation Coding Techniques", ACM International Conference on Advances in Computing, Communication and Control (ICAC3-2009), pp.: 384-390, 23-24 Jan 2009, Fr. Conceicao Rodrigous College of Engg., Mumbai. Available online at ACM portal.

[ 14 ] H.B.Kekre, Tanuja K. Sarode, Sudeep D. Thepade, "DCT Applied to Column mean and Row Mean Vectors of Image for Fingerprint Identification", International Conference on Computer Networks and Security, ICCNS-2008, 27-28 Sept 2008, Vishwakarma Institute of Technology, Pune.

[15] H.B.Kekre, sudeep thepade, archana athawale, anant shah,prathmesh velekar,suraj shirke, “ walsh transform over row mean column mean using image fragmentation and energy compaction for image retrieval",International journal of computer science and engineering (IJCSE), Vol.2.No.1,S2010,47-54.

[ 16] H.B.Kekre, Vinayak Bharadi, "Walsh Coefficients of the Horizontal \& Vertical Pixel Distribution of Signature Template", In Proc. of Int. Conference ICIP07, Bangalore University, Bangalore. 10-12 Aug 2007.

\section{BIOGRAPHIES}

Dr. H. B. Kekre has received B.E. (Hons.) in Telecomm. Engg. from Jabalpur University in 1958, M.Tech (Industrial Electronics) from IIT Bombay in 1960, M.S.Engg. (Electrical Engg.) from University of Ottawa in 1965 and Ph.D.(System Identification) from IIT Bombay in 1970. He has worked Over 35 years as Faculty and H.O.D. Computer science and Engg. At IIT Bombay. From last 13 years working as a professor in Dept. of Computer Engg. at Thadomal Shahani Engg. College, Mumbai. He is currently senior Professor working with Mukesh Patel School of Technology Management and Engineering, SVKM's NMIMS University vile parle west Mumbai. He has guided 17 PhD.s 150 M.E./M.Tech Projects and several B.E./B.Tech Projects. His areas of interest are Digital signal processing, Image Processing and computer networking. He has more than 300 papers in National/International Conferences/Journals to his credit. Recently nine students working under his guidance have received the best paper awards. Currently he is guiding 10 $\mathrm{PhD}$. Students. He is life member of ISTE and Fellow of IETE.

Dhirendra S.Mishra has received his BE (Computer Engg) degree from University of Mumbai in 2002.Completed his M.E. (Computer Engg) from Thadomal shahani Engg. College, Mumbai, University of Mumbai. He is PhD Research Scholar and working as Assistant Professor in Computer Engineering department of Mukesh Patel School of Technology Management and Engineering, SVKM's NMIMS University, Mumbai, INDIA. He is life member of Indian Society of Technical education (ISTE), Member of International association of computer science and technology (IACSIT), Singapore, Member of International association of Engineers (IAENG). His areas of interests are Image Processing, Operating systems, Information Storage and Management 